\title{
Exact analytic expression for a subset of fourth virial coefficients of polydisperse hard sphere mixtures
}

\author{
Ronald Blaak \\ FOM Institute for Atomic and Molecular Physics, Kruislaan 40\%, \\ 1098 SJ Amsterdam, The Netherlands.
}

(January 30, 2018)

\begin{abstract}
We derive an exact, analytic expression for the fourth virial coefficient of a system of polydisperse spheres under the constraint that the smallest sphere has a radius smaller than a given function of the radii of the three remaining particles.
\end{abstract}

\section{INTRODUCTION}

It is surprisingly difficult to calculate analytic expressions for virial coefficients. Even the exact calculation of the second virial coefficient is in general extremely difficult. If particles have no other interaction than hard core repulsion, things are slightly easier. In the isotropic phase, the second virial coefficient $B_{2}$ of two arbitrary, convex particles, $A$ and $B$, can be determined by [1]

$$
B_{2}(A, B)=\frac{1}{8 \pi}(V(A) G(B)+S(A) M(B)+M(A) S(B)+G(A) V(B))
$$

where only geometrical quantities of both particles are used: the volume $V$, the surface area $S$, and the integrals $M$ and $G$ over the mean respectively Gaussian curvature. For higher virial coefficients only approximations of a similar type are known [2].

The second and third virial coefficients of identical spheres are known analytically and are obtained after a straightforward calculation. The fourth virial coefficient, for which the analytic expression due to Boltzmann is also known [3], is much more difficult. Beyond the fourth virial coefficient only numerical data are available 《4. More recently, data of the fourth and fifth virial coefficients of binary mixtures have been published by Saija et al [5].

In this paper we will focus on hard spheres with different radii, for which until now, no analytic results are known beyond the third virial coefficient. In section III we show the results for the second and third virial coefficients. In section [II we derive the known results for the fourth virial coefficient. The main problem, the calculation of the complete star diagram, is derived in section IV together with an inequality, which has to be satisfied, in order for the given expression to be valid. In section $\nabla$ we finish by discussing our result and some concluding remarks. 


\section{SECOND AND THIRD VIRIAL COEFFICIENT}

The general expression for the $n$th virial coefficient $B_{n}$ of a gas with pairwise, additive interaction $\phi_{i j}$ between particles $i$ and $j$ is given in terms of Mayer $f_{i j}$ functions by

$$
B_{n}=\frac{1-n}{n !} \lim _{V \rightarrow \infty} V^{-1} \int \cdots \int V_{n} d \mathbf{r}_{1} \cdots d \mathbf{r}_{n}
$$

where $\mathbf{r}_{i}$ are the spatial coordinates of particle $i$, and $V$ is the volume accessible for the particles. $V_{n}$ is the sum over all labeled stars with $n$ points given by

$$
V_{n} \equiv \sum_{\left\{S_{n}\right\}} \prod_{i>j}^{n} f_{i j}
$$

and expressed in the Mayer $f$ functions, which are related to the interaction potential by

$$
f_{i j} \equiv \exp \left(-\beta \phi_{i j}\right)-1
$$

For hard particles the Mayer $f$ function reduces to

$$
f=\left\{\begin{array}{cl}
0 & \text { if no overlap } \\
-1 & \text { if overlap }
\end{array}\right.
$$

because the interaction potential is either zero or infinity in the case that particles do overlap, respectively do not overlap.

The second virial coefficient $B_{2}(A, B)$ for two particles $A$ and $B$, has only one contributing diagram, and is given by

$$
B_{2}(A, B)=\frac{1}{2} \bigcirc-\bigcirc=\frac{1}{2} \text { (A- B , }
$$

where we have explicitly labeled the diagram. We use diagrams to represent the different integrals. Integrations are over all possible positions of the different particles with one particle fixed. Each solid line represents an overlap between the connected particles, and ,hence, some constraint on the position vectors. The signs, which are determined by the Mayer functions, are, for convenience, immediately put in front of the diagrams. The diagram in $B_{2}$ is the volume from which the second particle is excluded, in order not to cause an overlap. For spheres with radii $A$ and $B$ this is simply the volume of a sphere with radius $A+B$, leading to the well known result

$$
B_{2}(A, B)=\frac{2}{3} \pi(A+B)^{3} .
$$

Also the third virial coefficient $B_{3}(A, B, C)$ of three particles $A, B$ and $C$ has only one contributing diagram

$$
B_{3}(A, B, C)=\frac{2}{3 !} \bigcirc=\frac{1}{3} \text { A }
$$


Only in the case of simple objects, e.g. spheres and discs, are analytic expressions for the third virial coefficient known.

In order to proceed we will assume that the radii of the three spheres $A, B$ and $C$ satisfy the inequality $A \geq B \geq C$. We position the biggest particle at the origin and particle $B$ on the positive $z$-axis. The integral corresponding to the diagram consists of two contributions. The first contribution comes from the case where $B$ is completely inside particle $A$, in which case particle $C$ is only required to overlap with particle $B$, leading automatically to an overlap with particle $A$. The second contribution comes from the case where $B$ is overlapping with $A$, but not enclosed. Particle $C$ now has to overlap with both particles, $A$ and $B$, and hence its center has to be placed in the overlapping volume of the spheres placed at $A$ with radius $A+C$ and the sphere placed at $B$ with radius $B+C$. This volume $Z\left(z_{A B}, A+C, B+C\right)$ is the sum of two segments of different spheres and depends on the distance $z_{A B}$ between $A$ and $B$. The formula $Z\left(r, R_{1}, R_{2}\right)$ can easily be evaluated if we assume $R_{1} \geq R_{2}$

$$
Z\left(r, R_{1}, R_{2}\right)=\left\{\begin{array}{l}
\frac{4 \pi}{3} R_{2}^{3} \quad r<R_{1}-R_{2} \\
\frac{\pi}{12 r}\left(R_{1}+R_{2}-r\right)^{2}\left(r^{2}-3\left(R_{1}-R_{2}\right)^{2}+2 r\left(R_{1}+R_{2}\right)\right) \\
0 \quad r>R_{1}+R_{2}
\end{array}\right.
$$

if $r<R_{1}-R_{2}$ the smaller sphere is completely enclosed by the larger sphere, and, hence, the overlap is equal to the volume of the smaller sphere. If $r>R_{1}+R_{2}$ the spheres are not overlapping at all. The diagram for the third virial coefficient is therefore given by

$$
\text { (B) }=\int_{0}^{A+B} d z_{A B} 4 \pi z_{A B}^{2} Z\left(z_{A B}, A+C, B+C\right)
$$

which results for the third virial coefficient $B_{3}(A, B, C)$ in

$$
\begin{array}{r}
B_{3}(A, B, C)=\frac{16 \pi^{2}}{27}\left(A^{3} B^{3}+B^{3} C^{3}+A^{3} C^{3}+\right. \\
3 A B C[A+B+C][A B+B C+C A]) .
\end{array}
$$

Although we have assumed that $A \geq B \geq C$, the resulting formula is fully symmetric in the radii.

\section{FOURTH VIRIAL COEFFICIENT}

In order to obtain the fourth virial coefficient $B_{4}$ for particles $A, B, C$ and $D$, we need to evaluate three different diagrams, although this could be reduced to only two modified star diagrams (see ref. \&])

$$
B_{4}(A, B, C, D)=-\frac{3}{4 !}(3 \mathrm{O}-6 \mathrm{O}+\mathrm{O}+\mathrm{O}) .
$$

The fourth virial coefficient is here expressed in unlabeled diagrams. However, labeling now becomes important because we want to evaluate those diagrams for the case of non-identical particles. This leads to the following expressions 


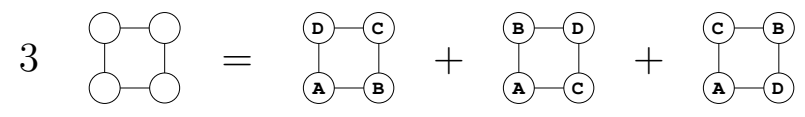

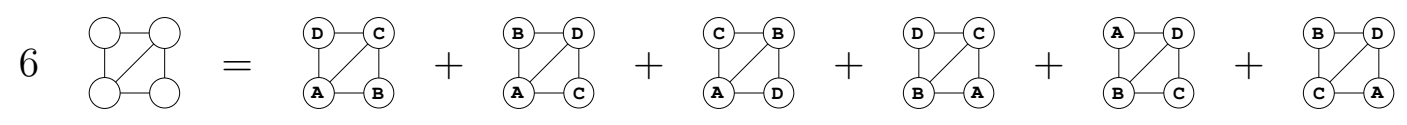

$$
\begin{aligned}
& 3 \operatorname{SO}^{-(D)}
\end{aligned}
$$

In order to proceed, we need to assume that the radii of the spheres satisfy $A \geq B \geq$ $C \geq D$. The labeled diagrams in the first line of (13) are given by expressions of the form

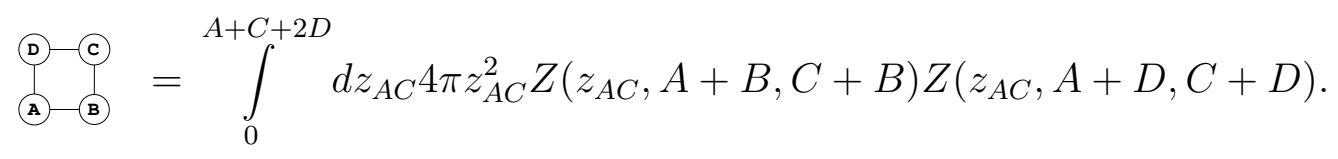

The limits of integration are a consequence of the differences in size and in this case determined by the fact that $B>D$. The diagrams in the second line of (13) are similar, and only differ in the integration limits because of the extra overlap. They are of the form

$$
\overbrace{\text { (D)- (B) }}^{\text {(D) }}=\int_{0}^{A+C} d z_{A C} 4 \pi z_{A C}^{2} Z\left(z_{A C}, A+B, C+B\right) Z\left(z_{A C}, A+D, C+D\right) \text {. }
$$

The other diagrams are obtained by permutation symmetry. The resulting integrals of (14) and (15) can easily be evaluated, but lead to lengthy expressions. The summations over the different labelings do not lead to symmetric expressions in terms of the radii. If we however take the combination of the diagrams according to the definition of the fourth virial coefficient (12), we obtain

$$
\begin{gathered}
3 \multimap-6 \bigcirc=-\frac{64 \pi^{3}}{9}\left(A^{3} B^{3} C^{3}+A^{3} B^{3} D^{3}+A^{3} C^{3} D^{3}+B^{3} C^{3} D^{3}+\right. \\
3 A B C D[A B+A C+B C+A D+B D+C D][A B C+A B D+A C D+B C D]),
\end{gathered}
$$

which is symmetric in the different radii of the spheres.

\section{THE COMPLETE STAR DIAGRAM}

The problem of calculating the fourth virial coefficient lies in the remaining diagram, the complete star diagram in which all pairs of the four particles overlap. An analytic expression for this diagram in general is not known. What is known is the special case of four identical radii, which is due to Boltzmann [3],9].

$$
\prod_{\mathrm{R}-\mathrm{R}}^{\mathrm{R}}=\frac{256 \pi^{2} R^{9}}{945}(3419 \pi-438 \sqrt{2}-8262 \arccos (1 / \sqrt{3})),
$$


where we used $R$ to denote the radius of the particles.

Some limiting cases for different radii are easily obtained. In the limit that the radius of the smallest sphere goes to zero the three remaining particles have to overlap with a point

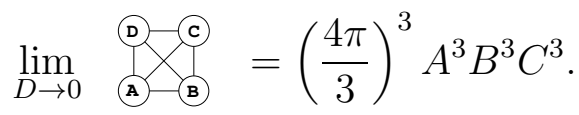

In the limit where the radius of the biggest sphere goes to infinity, the other three particles give rise to the diagram related to $B_{3}$

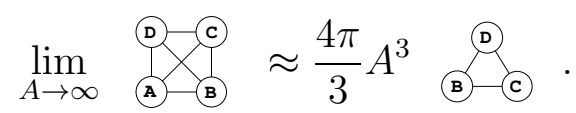

In the limit where both $A$ and $B$ go to infinity

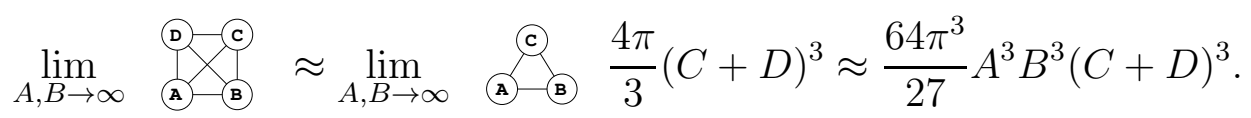

In order to explore the star diagram for non-limiting cases we must look in more detail to the diagrams and introduce the $\tilde{f}$-bonds as defined by Ree and Hoover 80 which we denote by a dashed line. Such a connection refers to non-overlapping particles, solid connections to overlapping particles and no connection allows for both, overlap and no overlap.

We will now consider diagrams in which the particles $A, B$ and $C$ all overlap with the smallest particle $D$. The diagram without other constraints can be written as the following summation of diagrams:

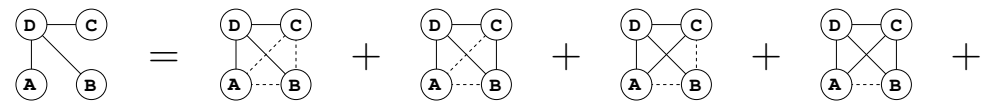

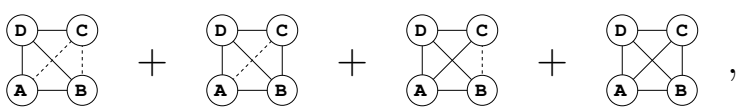

where we have denoted at the right hand side explicitly whether pairs of particles overlap or not. The diagram at the left hand side can, however, be evaluated immediately

$$
\int_{(\mathrm{D})}^{\mathrm{D}-\mathrm{C}}=\text { (A) } \mathrm{B} \times \text { B }- \text { (D) } \times \text { (C) - D } .
$$

The same is true for combinations of the form:

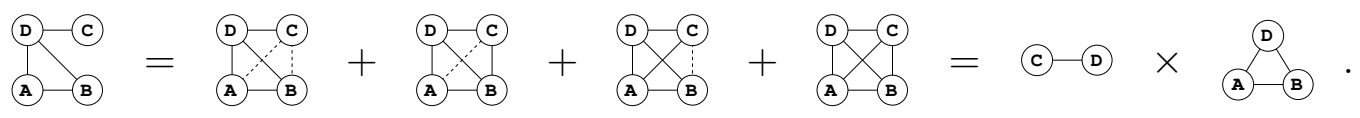


As well, some combinations have already been evaluated before (15), e.g.

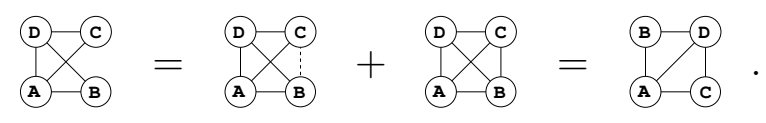

This leads to the following expression for the complete star diagram

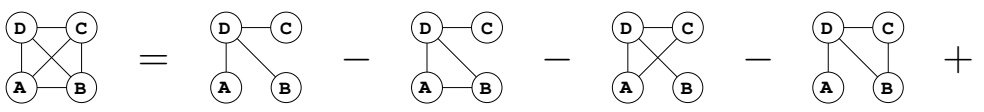

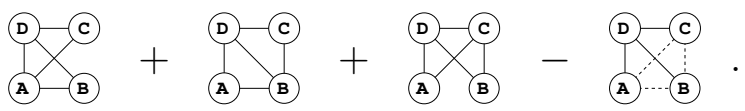

For all diagrams on the right hand side we have an analytic expression with only one exception - the last diagram. We demonstrate below that this last diagram is identical to zero if the radii of the spheres satisfy a simple inequality.

In order for this diagram to be non-zero the spheres with radius $A, B$ and $C$ should all overlap with the smallest sphere with radius $D$, but have no overlaps with each other. If the spheres $A, B$ and $C$ are mutually non-overlapping as shown in figure 1 , there is a smallest sphere $D$ that can be constructed that will touch with all three spheres. The centers of mass must all lie in the same plane, and therefore this problem reduces to the one of touching circles, known as Apollonian circles since the construction of this fourth circle was first solved by the Greek mathematician Apollonius of Perga (200 BC) [10]. If the radius $D$ were smaller, at least one of the spheres $A, B$ or $C$ would have no overlap with this circle.

If this were the case for all possible configurations of non-overlapping spheres $A, B$ and $C$ there would be no contribution to the last diagram of (25), and hence we would know the fourth virial coefficient. The smallest radius $D$ which can contribute is found in the case that all four spheres touch as depicted in figure 2. For this special case of the Apollonian problem, a simple relation between the radii of the circles was derived by the French philosopher and mathematician Descartes [10], known as the Descartes circle theorem:

$$
\left(\frac{1}{A}+\frac{1}{B}+\frac{1}{C}+\frac{1}{D}\right)^{2}=2\left(\frac{1}{A^{2}}+\frac{1}{B^{2}}+\frac{1}{C^{2}}+\frac{1}{D^{2}}\right) .
$$

Solving this equation gives the upper limit for radius $D$

$$
D \leq \frac{A B C}{A B+A C+B C+2 \sqrt{A B C(A+B+C)}},
$$

which will lead to a zero-valued diagram, and hence enables us to give an analytic expression for the complete star diagram. Therefore we obtain an analytic expression for the fourth virial coefficient of polydisperse spheres under the constraint (27)

$$
\begin{aligned}
& B_{4}(A, B, C, D)=\left(\frac{16 \pi^{3}}{27}\right) \times\left(A^{3} B^{3} C^{3}+A^{3} B^{3} D^{3}+A^{3} C^{3} D^{3}+B^{3} C^{3} D^{3}+\right. \\
& 3 A B C D[A B+A C+B C+A D+B D+C D][A B C+A B D+A C D+B C D])- \\
& \frac{16 \pi^{3}}{3} D^{3} A^{2} B^{2} C^{2}+\frac{8 \pi^{3}}{3} D^{4} A B C(A B+A C+B C)- \\
& \frac{8 \pi^{3}}{15} D^{5}\left(A^{2} B^{2}-2 A^{2} B C-2 A B^{2} C+A^{2} C^{2}-2 A B C^{2}+B^{2} C^{2}\right)- \\
& \frac{8 \pi^{3}}{5} D^{6}(A+B)(A+C)(B+C)-\frac{32 \pi^{3}}{35} D^{7}\left(A^{2}+3 A B+B^{2}+3 A C+3 B C+C^{2}\right)- \\
& \frac{8 \pi^{3}}{7} D^{8}(A+B+C)-\frac{8 \pi^{3}}{21} D^{9}
\end{aligned}
$$


The first part is symmetric in the four different radii and proportional to (16), the last part, however, is only symmetric in the radii $A, B$ and $C$. Note that the limiting cases (18), (19) and (20) are in agreement with this result.

In the case that $A=B=C=1$, and, since according to $(27) D \leq(2 \sqrt{3}-3) / 3 \approx 0.1547$, this formula reduces to

$$
\begin{aligned}
& B_{4}(1,1,1, D)=\left(\frac{8 \pi^{3}}{945}\right)\left(70+630 D+2520 D^{2}+1470 D^{3}+945 D^{4}+\right. \\
&\left.189 D^{5}-1512 D^{6}-1296 D^{7}-405 D^{8}-45 D^{9}\right) .
\end{aligned}
$$

This result can be compared with the numerical data for the binary mixture [7]. Only the first three numbers in the first column satisfy the inequality (27)

$$
\begin{aligned}
& D_{1112}\left(\mathcal{R}=\frac{1}{20}\right)=\frac{11058144323491 \pi^{3}}{6193152000000000}=0.0553630659 \quad[0.05539(2)] \\
& D_{1112}\left(\mathcal{R}=\frac{2}{20}\right)=\frac{31952948861 \pi^{3}}{12096000000000}=0.0819065785 \quad[0.08189(2)] \\
& D_{1112}\left(\mathcal{R}=\frac{3}{20}\right)=\frac{23207369313073 \pi^{3}}{6193152000000000}=0.1161886732 \quad[0.11617(3)]
\end{aligned}
$$

The numerical values obtained by Saija et al. [7] are given in square brackets.

\section{DISCUSSION}

To our knowledge this is the first time that an exact and analytic solution of the fourth virial coefficient of a hard sphere mixture is found. One should note, however, that in order for the expression to be valid, the radius of the smallest sphere is at most 0.1547 times that of the largest sphere. Our result agrees perfectly with the relevant, numerical results of Saija et al [7]. However, in the description of a polydisperse mixture of spheres, the most interesting case is that of a few large and many small particles. Unfortunately, this also means that the inequality (27) is, in general, not satisfied. The inequality requires that the smallest radius is strictly smaller than the other three, which, for instance in a binary

mixture, allows only one of the three mixed virial coefficients to be evaluated.

The method described in this article can almost certainly be used to evaluate the fourth virial coefficient of other particles, e.g. discs, and can possibly be used to simplify calculations of higher virial coefficients of asymmetric mixtures.

\section{ACKNOWLEDGEMENTS}

We thank Bela Mulder, José Cuesta and James Polson for a critical reading of the manuscript. The work of the FOM Institute is part of the research program of FOM and is made possible by financial support from the Netherlands Organization for Scientific Research (NWO). 


\section{REFERENCES}

[1] Isihara, A., 1950, J. Chem. Phys., 18, 1446.

[2] Boublík, T., and Nezbeda, I., 1986, Czech. chem. Commun. , 51, 2301.

[3] Boltzmann, L., 1899, Verslag Gewone Vergader. Afdel. Natuurk. Koninkl. Ned. Akad. Wetenschap, 7, 484.

[4] Kratky, K. W., 1977, Physica A, 87, 584.

[5] Saija, F., Fiumara, G., and Giaquinta, P. V., 1996, Mol. Phys., 87, 991.

[6] Saija, F., Fiumara, G., and Giaquinta, P. V., 1996, Mol. Phys., 89, 1181.

[7] Saija, F., Fiumara, G., and Giaquinta, P. V., 1997, Mol. Phys., 92, 1089.

[8] Ree, F. H., and Hoover, W. G., 1964, J. Chem. Phys., 40, 939.

[9] Happel, H., 1906, Ann. Physik, 21, 342.

[10] Coxeter, H. S. M., 1968, Amer. Math. Monthly, 75, 5. 


\section{FIGURE CAPTIONS}

1. The Apollonian problem of constructing a circle $D$ which is tangent to three arbitrary spheres $A, B$ and $C$.

2. A special case of the Apollonian problem in which all spheres are tangent to each other, and for which case a simple relation (26) between the radii was derived by Descartes. 


\section{FIGURES}

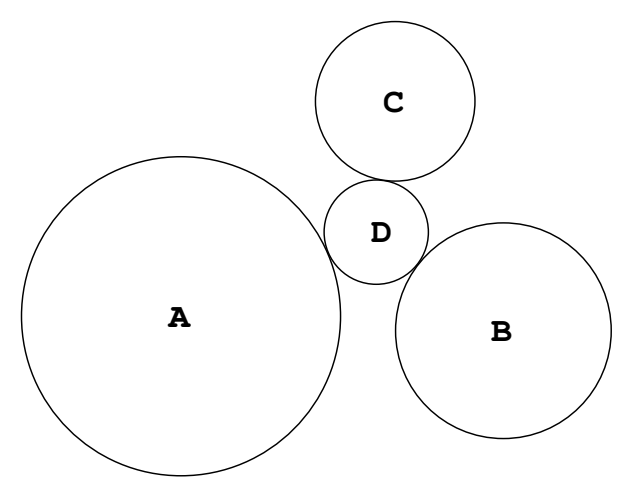

FIG. 1. Blaak

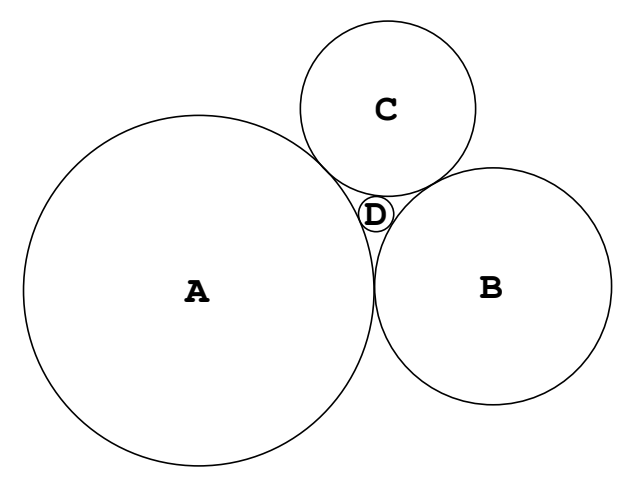

FIG. 2. Blaak 